\title{
Minute pulmonary meningothelial-like nodules: rare lesions appearing as diffuse ground-glass nodules with cyst-like morphology
}

\author{
Yawen Zhang ${ }^{1 \#}$, Jing $\mathrm{Wu}^{2 \#}$, Tao Zhang ${ }^{2}$, Qian Zhang ${ }^{3}$, Yu-Chen Chen ${ }^{2}$ \\ ${ }^{1}$ Department of Radiology, Nanjing PuKou Center Hospital, Nanjing, China; ${ }^{2}$ Department of Radiology, Nanjing First Hospital, Nanjing Medical \\ University, Nanjing, China; ${ }^{3}$ Department of Pathology, Nanjing First Hospital, Nanjing Medical University, Nanjing, China \\ \#These authors contributed equally to this work.
}

Correspondence to: Yu-Chen Chen. MD, PhD. Department of Radiology, Nanjing First Hospital, Nanjing Medical University, No.68, Changle Road, Nanjing 210006, China. Email: chenyuchen1989@126.com.

Submitted May 19, 2020. Accepted for publication Oct 27, 2020.

doi: 10.21037/qims-20-676

View this article at: http://dx.doi.org/10.21037/qims-20-676

\section{Introduction}

Minute pulmonary meningothelial-like nodules (MPMNs) are often discovered incidentally in surgical or autopsy specimens of the lung (1). They usually manifest as single or multiple lesions, and rarely present as diffuse on computed tomography (CT) images. In this case report, we describe a patient diagnosed with MPMNs, which appeared as diffuse ground-glass nodules (GGNs). We also retrospectively review the clinical, pathological, and CT characteristics of patient diagnosed MPMNs to help improve the understanding of the disease and the accuracy of diagnosis.

\section{Case presentation}

A 71-year-old female patient presented at our hospital in December 2016 with a cough that had lasted for 1 month. The patient had a history of coronary heart disease, diabetes, and hypertension, although these conditions were well controlled. She denied smoking, had no recent travel history, and tested negative for tuberculosis and antineutrophil cytoplasmic antibody (ANCA).

The CT scan showed diffuse GGNs in the bilateral lungs, and some of the nodules appeared to have a cystlike morphology. The nodules, measuring 2 to $5 \mathrm{~mm}$ in diameter with clear boundaries, were randomly distributed in the lungs, and had a tendency to be located in the peripheral zone, even on the interlobular fissure (Figure 1). The CT images showed observable blood vessels on the edges of some nodules. The patient underwent two re-examinations, in January 2018 and July 2019, and no changes were observed in the lesions. Because the nodules were discovered on interlobular fissures, disseminated lung disease was ruled out. According to these manifestations, a primary disease of the lung was likely. The results of the three subsequent reviews showed no changes. Subsequently, the patient was initially diagnosed with a benign lung tumor or early adenocarcinoma.

The patient underwent thoracoscopic partial wedge biopsy. We fixed the obtained surgical specimens in $4 \%$ neutral formaldehyde and embedded them in paraffin, stained with hematoxylin and eosin, then we observed the histological features of the lesions under an optical microscope. When surgical specimens observed at low magnification, the pulmonary biopsies showed multiple small well-defined meningothelial-like nodules formed by thickened alveolar walls. When surgical specimens observed at high magnification, these nodules were composed of oval to short spindle epithelioid cells with eosinophilic cytoplasms and mild nuclei, with indistinct cellular borders. Some of the epithelioid cells were arranged in a whorled pattern (Figure 2). Immunohistochemistry revealed that the cells were positive for epithelial membrane antigen (EMA), 

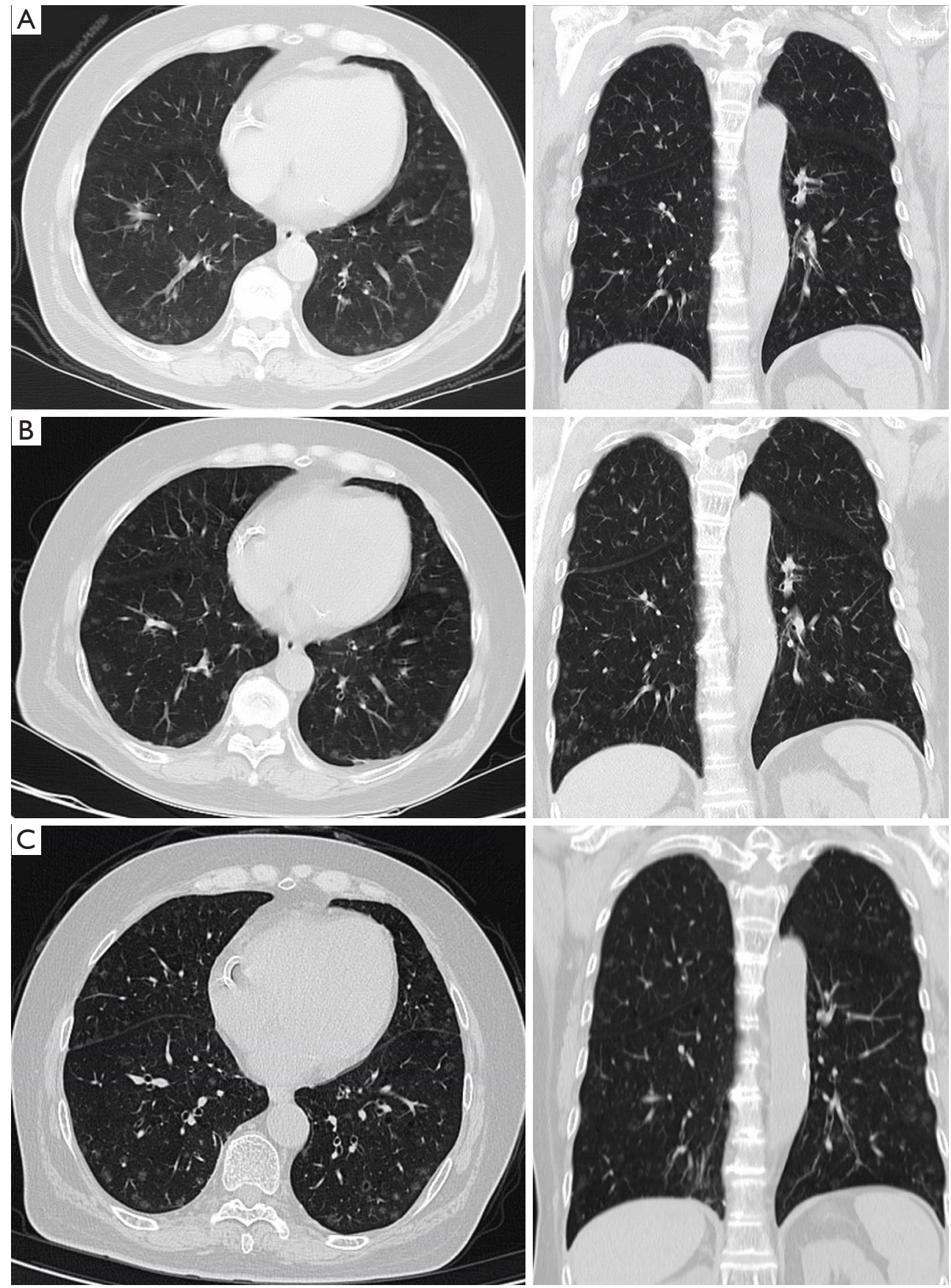

Figure 1 Coronary and axial chest computed tomography showed diffuse ground-glass nodules in bilateral lungs with the central cystic lesion in (A) December 2016, (B) January 2018, and (C) July 2019.

vimentin, progesterone receptor (PR), and CD56 (Figure 3), and negative for thyroid transcription factor 1 (TTF-1), S-100 protein, androgen receptor (AR), smooth muscle actin, desmin, and HMB-45. Finally, a diagnosis of MPMN was made.

\section{Discussion}

MPMNs were first described by Korn et al. (2) in 1960, although the exact origin and nature of these nodules remain controversial to this day. Because MPMNs have similar 


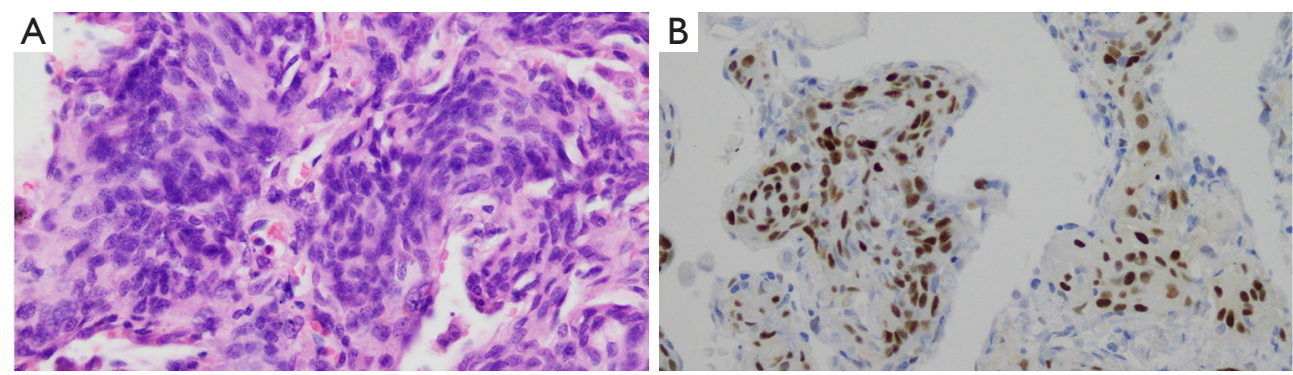

Figure 2 Histological staining. (A) Pulmonary biopsies showed epithelioid cells arranged in a whorled pattern (H\&E staining, $\times 400)$. (B) Immunohistochemistry for the progesterone receptor showed positive nuclear staining of meningothelial cells $(\times 200)$.
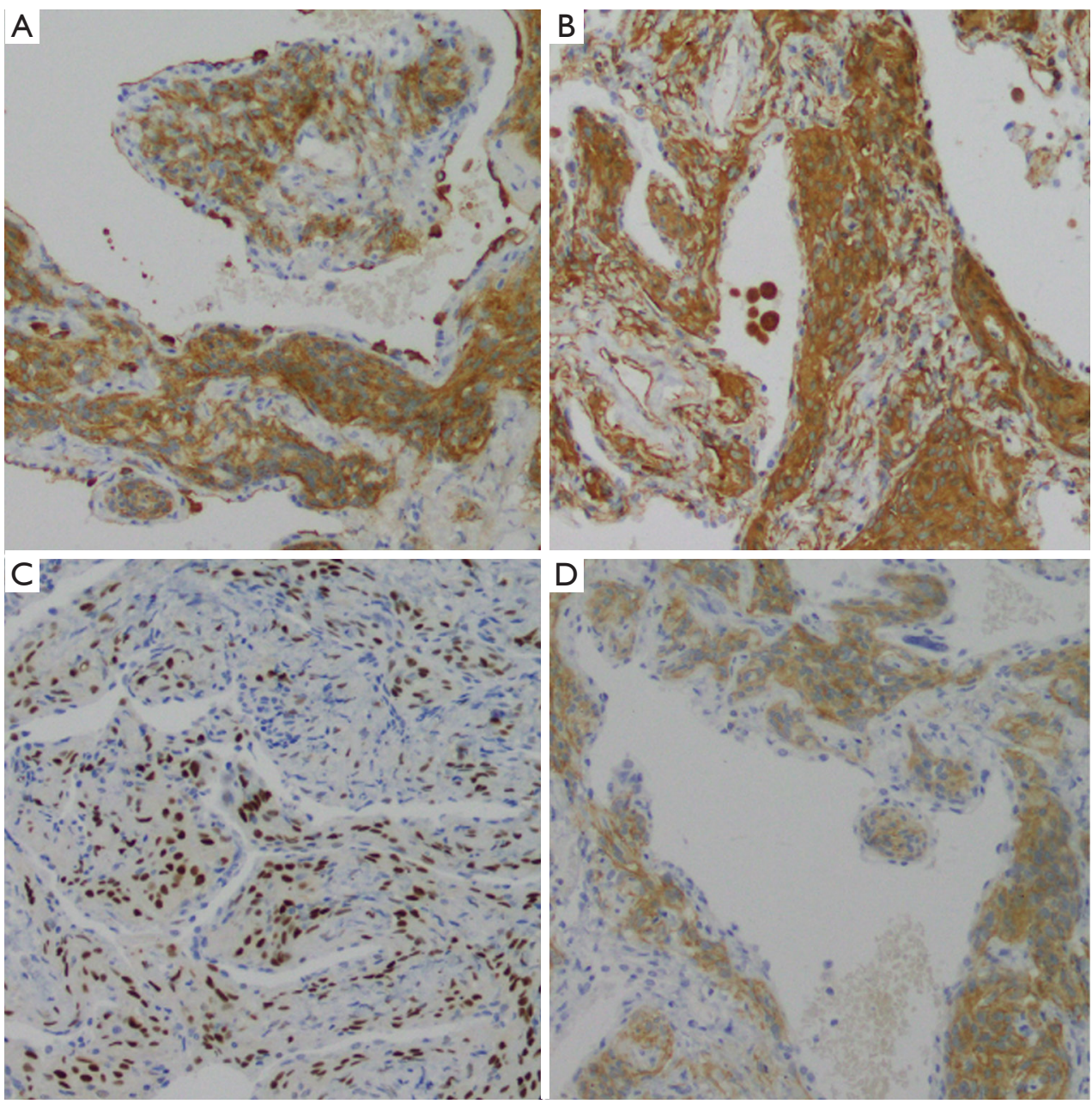

Figure 3 Immunohistochemical staining showed that cells were positive for (A) epithelial membrane antigen (EMA, $\times 200)$; (B) vimentin (×200), (C) progesterone receptor (×200), and (D) CD56 (×200).

histological, ultrastructural, and immunohistochemical features as meningiomas, including pleural or pulmonary meningiomas, an association between these two conditions has been suggested. Masago et al. reported that primary pulmonary meningioma presents as a giant form of MPMN, as determined by the immunohistochemical findings, 
including positive PR and CD56 staining (3). However, genetic analysis of these structures indicate a reactive rather than a neoplastic origin (4). Accordingly, the new term "meningothelial-like nodule" was proposed by Gaffey et al. (5), and appeared as "minute meningothelial nodule" in the third edition of the World Health Organization International Histological Classification of Tumors (6). When the nodules are diffusely distributed in the bilateral lungs. We will diagnose them as diffuse pulmonary meningotheliomatosis (DPM) or MPMN-omatosis (7).

According to the observations made in this case study, under a low-power microscope, multiple lesions could be observed in one field of view, and the lesions could be outlined so that they appeared with clear boundaries on the image. Nodules could be close to the pleura but will not invade the pleura. At high magnification, round, oval, or fusiform meningeal epithelial cells could be seen in a nested distribution. According to our observations, the lesion originated from the alveolar interstitium rather than the alveolar epithelial tissue. Therefore, the thickening of the alveolar wall as well as the air in the alveolar cavity caused some of the nodules to appear with a cyst-like morphology. The CT images showed blood vessels on the edges of some nodules. However, whether nodule and blood vessel are related is still unknown. The vessel wall was smooth and the thickness of the lumen was uniform, suggesting that the lesion may grow inertly and non-invasively. In the three examinations before and after the operation, no new nodules or enlarged nodules were found. The immunohistochemical results of the data in this case show that the lesion do not originate from alveolar epithelium. This is different from adenocarcinoma, which originate from alveolar epithelium.

Many diseases appear similar to diffuse cystic micronodules, including multiple adenocarcinomas, pulmonary Langerhans cell histiocytosis (PLCH), and multifocal micronodular pneumocyte hyperplasia (MMPH) (8). However, PLCH usually occurs in young men with a history of smoking, and PLCH lesions are mostly located in the upper lobe of the bilateral lungs and generally do not involve the pleura, which does not match the CT findings in this case. MMPH occurs in patients with tuberous sclerosis. By combining the patient's medical history with laboratory test results, we ruled out the possibility of these two diseases. However, we could not exclude the possibility of multiple adenocarcinomas based on the CT image. Interestingly, according to some relevant literature, multiple MPMNs may be associated with several concomitant diseases, such as pulmonary thromboembolism, smoking-related interstitial lung disease, atypical adenomatous hyperplasia, and cardiac disease. However, these correlations between have not been confirmed. MPMNs are observed more frequently in females, this conclusion is consistent with the result reported in the literature.

Although it is extremely difficult to diagnose MPMNs only from images, we have reviewed some relevant literature and summarized the image representations of MPMNs. Most MPMNs are small (1 to $3 \mathrm{~mm}$ in diameter) and are randomly distributed throughout the bilateral lungs, and the lesions have a tendency to be located in the peripheral zone, even on the interlobar pleural (6). MPMNs have clear boundaries, and the lesions can appear as single, multiple, or diffuse nodules. The CT scan often shows GGNs in the bilateral lungs.

In summary, MPMNs are a rare occurrence and have unique imaging characteristics. When observing diffuse GGNs, as in the case described here, attention should be paid to the possibility of MPMNs.

\section{Acknowledgments}

Funding: None.

\section{Footnote}

Conflicts of Interest: All authors have completed the ICMJE uniform disclosure form (available at http://dx.doi. org/10.21037/qims-20-676). The authors have no conflicts of interest to declare.

Ethical Statement: Written informed consent was obtained from the patient for publication of this study and any accompanying images.

Open Access Statement: This is an Open Access article distributed in accordance with the Creative Commons Attribution-NonCommercial-NoDerivs 4.0 International License (CC BY-NC-ND 4.0), which permits the noncommercial replication and distribution of the article with the strict proviso that no changes or edits are made and the original work is properly cited (including links to both the formal publication through the relevant DOI and the license). See: https://creativecommons.org/licenses/by-nc-nd/4.0/.

\section{References}

1. Kuroki M, Nakata H, Masuda T, Hashiguchi N, Tamura 
S, Nabeshima K, Matsuzaki Y, Onitsuka T. Minute pulmonary meningothelial-like nodules: high-resolution computed tomography and pathologic correlations. J Thorac Imaging 2002;17:227-9.

2. Korn D, Bensch K, Liebow AA, Castleman B. Multiple minute pulmonary tumors resembling chemodectomas. Am J Pathol 1960;37:641-72.

3. Masago K, Hosada W, Sasaki E, Murakami Y, Sugano M, Nagasaka T, Yamada M, Yatabe Y. Is primary pulmonary meningioma a giant form of a meningothelial-like nodule? A case report and review of the literature. Case Rep Oncol 2012;5:471-8.

4. Kraushaar G, Ajlan AM, English JC, Müller NL. Minute pulmonary meningothelial-like nodules: a case of incidentally detected diffuse cystic micronodules on thinsection computed tomography. J Comput Assist Tomogr 2010;34:780-2.

Cite this article as: Zhang $\mathrm{Y}, \mathrm{Wu} \mathrm{J}$, Zhang T, Zhang Q, Chen YC. Minute pulmonary meningothelial-like nodules: rare lesions appearing as diffuse ground-glass nodules with cyst-like morphology. Quant Imaging Med Surg 2021;11(7):3355-3359. doi: 10.21037/qims-20-676
5. Gaffey MJ, Mills SE, Askin FB. Minute pulmonary meningothelial-like nodules. A clinicopathologic study of so-called minute pulmonary chemodectoma. Am J Surg Pathol 1988;12:167-75.

6. Travis WD, Colby TV, Corrin B, Shimosato Y, Brambilla E. Histological classification of lung and pleural tumors. WHO International Histological Classification of Tumors: Histological Typing of Lung and Pleural Tumors. Springer, 1999:21-66.

7. Huang EC, Zhang Y, Bishop JW, Gandour-Edwards RF, Afify AM. Diffuse pulmonary meningotheliomatosis: A diagnostically challenging entity on fine-needle aspiration cytology. Diagn Cytopathol 2015;43:727-30.

8. Mizutani E, Tsuta K, Maeshima AM, Asamura H, Matsuno Y. Minute pulmonary meningothelial-like nodules: clinicopathologic analysis of 121 patients. Hum Pathol 2009;40:678-82. 